\title{
QoS enhancement in MANETs using priority aware mechanism in DSR protocol
}

\author{
Jayson K. Jayabarathan ${ }^{1 *}$, Sivanantha Raja Avaninathan ${ }^{2}$ and Robinson Savarimuthu ${ }^{1}$
}

\begin{abstract}
Quality of service (QoS) enhancement is one of the prominent research areas in a mobile ad hoc network in which a variety of routing protocols is introduced. However, those routing protocols do not support efficiently, the multiple routes in the network, as congestion occurs owing to multiple routes. Hence, the infrastructure-less and dynamic nature of the mobile ad hoc network (MANET) demands a new set of networking strategies to improve the QoS. In this paper, a priority aware (PA) mechanism is implemented by assigning priority in accordance with their data rates in dynamic source routing (DSR) protocol in order to enhance the QoS for MANET. Five different connections are considered, and the performance parameter of the network such as throughput, packet delivery ratio (PDR), and end-to-end delay (EED) is estimated for the proposed priority aware DSR (PA-DSR) protocol. From the simulation, it is observed that the proposed PA-DSR protocol performs better than the DSR protocol due to its rate cutting mechanism.
\end{abstract}

Keywords: Quality of service, Bandwidth estimation, Ad hoc on-demand distance vector, Priority aware mechanism, Mobile ad hoc networks, DSR protocol

\section{Introduction}

A mobile ad hoc network (MANET) is a self-starting dynamic network, comprising of mobile nodes, where all the participating nodes are voluntarily transmitting the packets from one place to another place and assumed to be dynamic with more or less relative speed in an arbitrary direction [1-4]. Hence, it is highly difficult to ensure the long-term guaranteed path from one node to the other node. Typically, the MANET is employed for emergency scenarios like military operations, monitoring animal habitats, and disaster relief operation where there is a need for communication network immediately following some major event or some temporary requirement like a conference or seminar at a new place where no earlier network infrastructure exist and an alternative solution is needed [5-7].

The emergence of real-time applications and the widespread utilization of wireless and mobile devices have generated the need to provide quality of service (QoS) support in wireless and mobile networking environments.

\footnotetext{
* Correspondence: jaysonmzcet@gmail.com

${ }^{1}$ Mount Zion College of Engineering and Technology, Pudukkottai-622507,

Tamil Nadu, India

Full list of author information is available at the end of the article
}

It is very important to determine the QoS of the network which is primarily depending upon the network. In MANET, there are several parameters which influence enhancing the QoS of the network such as throughput, end-to-end delay, packet delivery ratio, and jitter $[8,9]$. The aforementioned parameters are improved by altering the algorithm, protocol, and mechanisms.

Typically, QoS refers to the ability of a network to provide improved service to selected network traffic over various underlying technologies [8]. QoS routing requires finding not only a route from a source to a destination but also a route that satisfies the end-to-end QoS requirement. QoS is more difficult to guarantee in ad hoc networks than in most other types of networks, because the wireless bandwidth is shared among adjacent nodes and the network topology changes owing to the movement of nodes. Hence, in order to provide QoS in MANET, the extensive collaboration between nodes is essential to establish the route and to secure the resources $[10,11]$. Primarily, QoS can be achieved by two ways: (a) overprovisioning and (b) traffic engineering. Over-provisioning employs the best-effort approach and simply increases the available resources. Alternatively, traffic engineering tries to exploit resources efficiently and to make the network 
QoS aware which includes additional service classes, admission control, and resource reservations [12].

QoS provisioning improves the end-to-end performance in heavily loaded networks through QoS aware routing, admission control, resource reservation, traffic analysis, and scheduling [12]. The goal of QoS provisioning is to achieve more deterministic network behaviors, where in turn, the information carried by the network can be delivered accurately and network resources can be utilized properly. However, there still remains a significant challenge to provide QoS solutions and maintain end-to-end QoS with user mobility. QoS provisioning will lead to an increase in computational and communicational cost. The QoS provisioning approaches can be classified into two categories: hard QoS and soft QoS approaches. If QoS requirements of a connection are guaranteed to be met for the whole duration of the session, the QoS approach is termed as hard QoS approach. In MANETs, it is very challenging to provide hard QoS guarantees to user applications. In soft QoS, the Qos requirements are not guaranteed for the entire session [12]. There are several challenges that are reported while providing Qos in MANETs such as hidden terminal problem, lack of central coordination, insecure medium, limited resource availability, dynamically varying network topology, error-prone shared radio channel, and imprecise state of information. In MANETs, one of the most crucial components of a system for QoS provisioning is to estimate the state of the network resources and thereby decide which application data can be processed. To estimate the available bandwidth in a heavily loaded wireless network is a non-trivial task due to the aforementioned factors of wireless networks [13].

In the literature, there are several non-linear programming methods such as genetic algorithm, fuzzy logic, and neural network which are employed to find the feasible routes in order to improve the QoS. The QoS of MANETs is enhanced using computational intelligent techniques [14], fuzzy multi-objective routing [15], flooding limited and multiconstrained multicast routing using genetic algorithm [16], multicast protocol (codepipe) [17, 18], optimize congestion and dilation [19], physarum optimization for long stating computational problems (Steiner tree problem) with low complexity and high parallelism [20], biology-based algorithm [21], interference-based topology control algorithm [22], spatial reusability aware routing [23], etc.

In MANET, there is no reliable mechanism to provide QoS; therefore, research in this field has received much attention from the last decade. Though there is no QoS mechanism for IEEE 802.11-based MANETs, it can provide some QoS level through service differentiation, due to the IEEE 802.11e amendment. However, no solution has been standardized for estimation of bandwidth which becomes necessary for guaranteed QoS. The estimation of available resources still represents one of the main issues for QoS enhancement. Hence, the bandwidth estimation must be accurate enough to assure the admission of right connections.

In this paper, a priority aware dynamic source routing (DSR) is proposed to enhance the QoS by estimating the available bandwidth in IEEE 802.11-based MANET. Five connections are considered, and their priority is assigned according to its data rates. The QoS parameters throughput, packet delivery ratio, and end-to-end delay are estimated with respect to the simulation time and total number of nodes in the network with and without mobility.

This paper is organized as follows: The reported bandwidth estimation techniques to enhance the QoS are presented in Section 2. The proposed priority aware DSR protocol is given in Section 3. The simulation results of priority aware DSR (PA-DSR) such as the effect of throughput, packet delivery ratio, and end-to-end delay with respect to the simulation time and nodes are discussed in Section 4. Finally, Section 5 concludes the paper.

\section{DSR protocol}

DSR protocol is a routing protocol for MANET where no infrastructure is required. The multihop nature in DSR enables nodes to forward packets to neighboring nodes when the nodes are within the transmission range. The originator (sender) of the data initiates route discovery whenever the location of the destined receiver is not known. The route discovery packet, i.e., route request (RREQ), is flooded. Upon reaching every node, each node can read the packet information such as sender address, destination address, and request ID determined by the originator. Further, each node appends its identity when forwarding RREQ to the next nodes. Once the information has reached the target node, it sends a route replay message to the originator. Further, the entire route recorded in the RREQ packet is copied to route reply (RREP) and forwarded back to the originator. The originator upon receiving this information caches the route for subsequent routing. The intermediate nodes discard any packet when the packet contains the same ID that it has seen before or it finds its address in the recorded route; otherwise, it appends its address to route record in the RREQ packet $[24,25]$.

Whenever the topology has changed or a link to the respondent broken, the originator of the packet can sense it and route maintenance indicates that the source route is broken. Now, the originator of the packet can choose any other route to reach its respondent or it can initiate a new route discovery. The route maintenance is used when the originator send packets to its recipient. The on-demand nature of route request and route maintenance 
requires no periodic routing advertisement or link sensing mechanism. Due to the lack of periodic advertisement, there are no overhead packets caused by DSR. A response to single route discovery enables the node to learn and cache multiple routes to any destination. This mechanism enables any node to react quickly whenever link failure occurs $[24,25]$.

\section{Methodology}

\subsection{Related work}

In MANET, estimation of available bandwidth is a primary component for QoS. The available bandwidth refers to the amount of bandwidth available to the node to send packets to the network. The available bandwidth is employed for analyzing the network performance and optimizing end-to-end transport performance which is used to improve the QoS of multimedia services and video streaming over a network as such applications require large bandwidth [13]. Typically, the bandwidth estimation is classified into four different categories: (a) active probing techniques, (b) passive techniques, (c) techniques only for wireless networks, and (d) other bandwidth estimation techniques [13].

In the literature, there have been several passive techniques reported to estimate the available bandwidth which in turn improve the QoS. The bandwidth estimation using passive technique is the calculation-based technique where the available bandwidth is estimated using the measured channel usage without any impact on the existing flows. De Renesse et al. proposed to calculate the available bandwidth using the ratio between the numbers of transmitted and received packets. The available bandwidth is enhanced as it is calculated by minimizing the unnecessary signaling and stopping the sessions that cannot meet the QoS requirement. Hence, the message overhead is decreased while increasing the data rate [26]. The condense aware admission control protocol provides an efficient, scalable admission control protocol for the MANET to ensure the end-to-end connections with QoS requirements. The abovementioned mechanism offers a better QoS guarantee by limiting the number of flows (connections) in the network [27]. However, a node must have enough bandwidth to admit a flow successfully.

The adaptive admission control is estimated end-toend with the available bandwidth based on cross-layer QoS routing. It also considered the intraflow contention problem [28]. It assumes that channel idle period between the sender and the receiver is totally overlapped. The assumption overestimates the available bandwidth. Zhao et al. proposed the mechanism to calculate the available bandwidth in the particular link to support QoS in IEEE 802.11-based networks. They have considered synchronization between transmitter and receiver in order to identify the bandwidth in a decided link [29]. It accurately estimates the existing bandwidth on a given link. The bandwidth estimation is carried out by an agent-based mechanism by Sabojil and Akki which enhances the latency and accuracy [30]. The optimum available bandwidth is calculated using static and mobile agent for a connectionless and connection-oriented network.

By using channel monitoring, collision estimation and backoff duration prediction and the available bandwidth are calculated [31]. When the nodes estimate the bandwidth, they collect the network allocation vector information and backoff duration from their neighbor nodes to compute the total busy period for the channel within the monitoring period. Chen and Heinzelman calculated the residual bandwidth of the IEEE 802.11 MAC where the bandwidth is shared among neighboring hosts [32]. The available bandwidth is estimated by combining the listen available bandwidth with the hello available bandwidth method. In a similar way, the available bandwidth is estimated according to the channel conditions like busy time (listen method) and hello method. Here, the bandwidth estimation is done by switching the channel conditions according to the channel state [33]. Sarr et al. presented a new technique to compute available bandwidth between two neighbor nodes and by extension along a path. The available bandwidth is calculated by collecting/exchanging the information during the communication among neighbor nodes without any impact on the other existing flows. It considered the collision probability that the packet undergoes. By calculating the bandwidth estimation techniques using carrier sensing mechanism, the communication is initiated with five different connections [34]. Though several methods are reported to estimate the available bandwidth, there is no such attempt made to assign the available bandwidth according to the priority-based applications (high data rate, emergency applications have the highest priority). The authors proposed a priority aware mechanism to estimate the available bandwidth, and different priorities are implemented among the connections according to the data rates with respect to the available bandwidth.

\subsection{Proposed work: priority aware DSR}

When congestion occurs in the network, the low priority packets travel faster than the higher priority packets. Hence, the packet dropping is much higher for lower priority packets than for higher priority packets. There is no priority fairness during the packet dropping condition which directly affects the overall throughput and delay in the network. Therefore, the reported priority mechanism in DSR diminishes the total QoS in the network. In order to enhance the QoS in the MANET, a new priority aware DSR protocol is proposed. 
In PA-DSR, the priority is assigned externally (user-defined/application-based) according to their requirements (data rate). In this proposed method, the rate cutting mechanism is deployed based on the threshold value, priority information, and transmission rate. Whenever the cumulative sum of the transmission rate field of communication exceeds the threshold limit, the low priority packet communication is routing down (not able to transfer). If the cumulative sum of the transmission rate does not exceed the threshold limit, the communication among the nodes is initiated for all the connections without any degradation of service. The best effort mechanism ensures more or less constant throughput, high packet delivery ratio, and low end-to-end delay which in turn ensure higher quality of service.

The proposed method contains three steps which are (a) threshold setting, (b) priority assignment, and (c) transmission rate

- Threshold setting

$\circ$ The maximum achievable throughput with constant end-to-end delay over the simulation period using DSR under no interference and no motion (one communication, i.e., one source and one destination) is around 85,000 bytes per second. The maximum data rate of 85,000 bytes are optimized through simulation in order to reduce the delay. Since the entire payload packets are in the size of 1000 bytes, the threshold value is converted into a round off value of 85 packets per second. This acts as a rate cutting mechanism according to its data rate.

- Priority assignment

○ All communications follow certain norms in mentioning their priority, i.e., no communication are greedy to tell that its priority is always high. Every communication tells a distinct priority number from 1 to 5 . The priority is assigned based on the data rates. The high data rate connection is designated as higher priority and vice versa.

- Transmission rate

$\circ$ There are two additional information added to every packet

- Priority

- Estimated transmission rate

- The transmission rate for every communication is presented in transmission rate field and every data packet. Its values are said in terms of packets per second, typically, 1, 20, 20,30, and 40 packets per second for communication (flow) 5, 4, 2, 3, and 1 , respectively.

If the cumulative sum of the transmission rate is greater than or equal to the threshold limit ( 85 packets
Table 1 Connections, flow id, assigned priority, data rate, and simulation time of PA-DSR

\begin{tabular}{llllll}
\hline Connections & $\begin{array}{l}\text { Flow } \\
\text { id }\end{array}$ & Priority & $\begin{array}{l}\text { Packets send } \\
\text { per second }\end{array}$ & $\begin{array}{l}\text { Starting } \\
\text { time }\end{array}$ & $\begin{array}{l}\text { Ending } \\
\text { time }\end{array}$ \\
\hline C1 & 3 & 3 & 30 & 2 & 50 \\
C2 & 2 & 2 & 20 & 10 & 50 \\
C3 & 1 & 1 & 40 & 20 & 50 \\
C4 & 5 & 5 & 1 & 30 & 50 \\
C5 & 4 & 4 & 20 & 40 & 59 \\
\hline
\end{tabular}

per second), then the low priority packet (flow id 5, flow id 4 , and so on) is not be able to make the connection, else the packets are transferred from source to destination without any delay. During the data transfer high throughput, high packet delivery ratio can be achieved.

The priority is assigned based on the data rate, i.e., the highest data rate is considered as the highest priority where a packet contains 1000 bytes. In the proposed PADSR, the highest priority is assigned for communication 3 (C3) as it has a high data rate and the low priority is designated for connection 5 (C5). The flow ids, assigned priority with its simulation time, and the assigned data rate for each flow are reported in Table 1.

Table 2 Simulation parameters of PA-DSR

\begin{tabular}{lll}
\hline Sl. no. & Parameters & Values \\
\hline 1 & Simulator & NS-2 (version 2.35) \\
2 & Simulation time & $60 \mathrm{~s}$ \\
3 & Topology area & $500 \mathrm{~m} \times 500 \mathrm{~m}$ \\
4 & Number of nodes & $20,40,60,80$, and 100 \\
5 & Mobility speed & 2 to $20 \mathrm{~m} / \mathrm{s}$ \\
6 & Mobility model & Random initial position \\
7 & Transmission range & and random motion \\
8 & Packet rate & $250 \mathrm{~m}$ \\
9 & Packet size & $8-320$ kbps \\
10 & Traffic type & 1000 bytes \\
11 & Number of CBR connections & Constant bit rate (CBR) \\
12 & Pause time & $0 \mathrm{~s}$ \\
13 & Channel type & Channel/wireless channel \\
14 & Antenna type & Antenna/omni antenna \\
15 & Interface queue type & Queue/drop tail/pri queue \\
16 & Protocol studied & DSR \\
17 & MAC type & IEEE 802.11 \\
18 & Frequency & 2.4 GHz \\
19 & Trace format & New wireless format \\
20 & Link layer type & Link layer \\
\hline
\end{tabular}




\section{Simulation results and discussion}

The QoS performance of DSR and PA-DSR routing protocol is analyzed with simulations which are implemented with NS2.35. The implementation is based on the DSR module. To illustrate the accurate estimation of PA-DSR, initially, a simulation is performed in a $500 \mathrm{~m} \times 500 \mathrm{~m}$ static network with 100 nodes. The nodes are randomly positioned five flows with different data rates (connections) which are attempted to be established in the network. The channel capacity is set to $1 \mathrm{Mbps}$, and five one-hop connections are established in the network. For each flow, the source is randomly chosen among the sources neighbors. Simulations are stopped after the 60th second. For each scenario, the results presented here are obtained over 30 simulation runs with different random seeds. The simulation parameters are listed in Table 2. In this simulation, quality of service evaluation is carried out by calculating the throughput, end-to-end delay, and packet delivery ratio.

Figure 1a, b represents the throughput evaluation of the five flows for DSR (when no admission control is incorporated) and PA-DSR, respectively. From Fig. 1a, it is observed that the obtained throughput of the network becomes congested for all the connections after the 20th second as there is no admission control mechanism available in the DSR protocol. This indicates that the network is not able to sustain this traffic scenario. As a consequence, the throughputs achieved by the flows are degraded. However, when the priority is assigned in DSR as shown in Fig. 1b, the throughput of the network for all the five flows is stable which represents all the admitted flows that are able to fit into the network. In Fig. 1b, it is noticed that PA-DSR accepts all the connections without any degradation. Therefore, the proposed admission control mechanism is very useful to allow all the users according to their priority without any collision. In addition, it is observed that in Fig. 1b, the communication 1 ( $\mathrm{C} 1 \rightarrow$ flow id 3 ) is started at the 2 nd second and routed down at the 20th second (before its assigned period) as the threshold limit of the network is reached at the 20th second (once C3 is initiated at the 20th second); the next priority packets are routed down (C1). However, after the 20th second, all the communications are ensured without any degradation of service, because the total capacity of the network is well below the threshold limit.

The conventional DSR protocol follows the worst scheduling case and underestimates the available bandwidth; hence, the throughput of the network is diminished once the network has reached its maximum capacity which in turn reduces the QoS. Alternatively, Fig. 1b shows the throughput achieved by PA-DSR which performs a

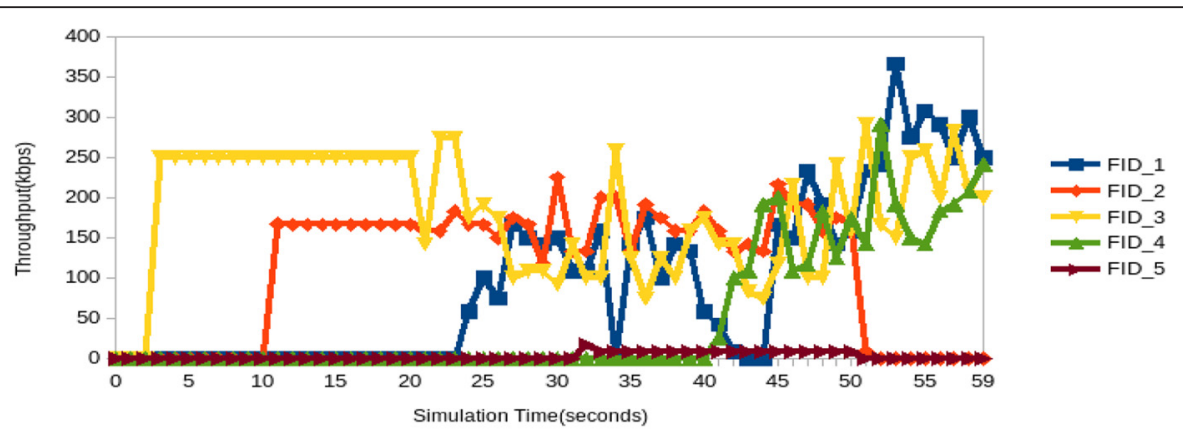

(a)

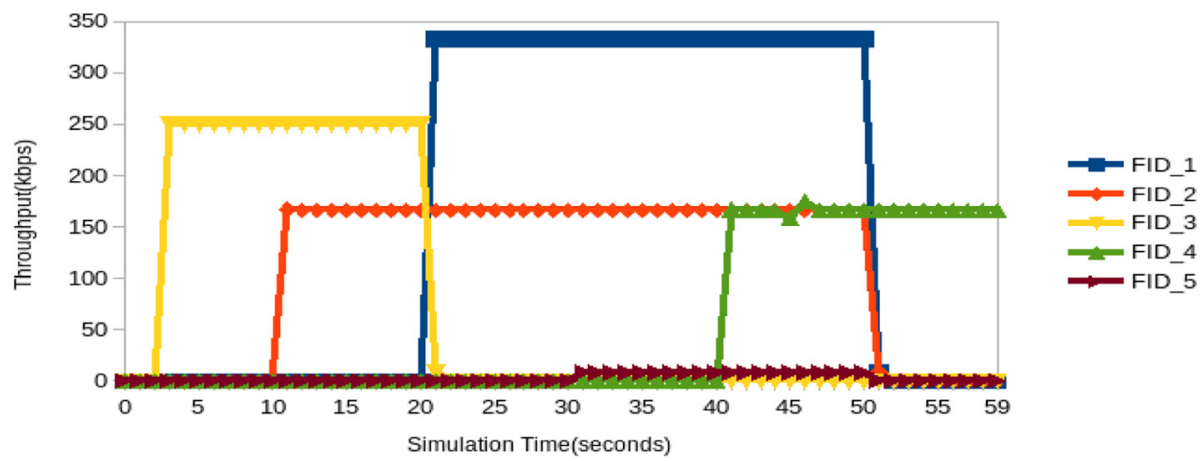

(b)

Fig. 1 Schematic representation of throughput vs simulation time for a DSR and $\mathbf{b}$ PA-DSR 


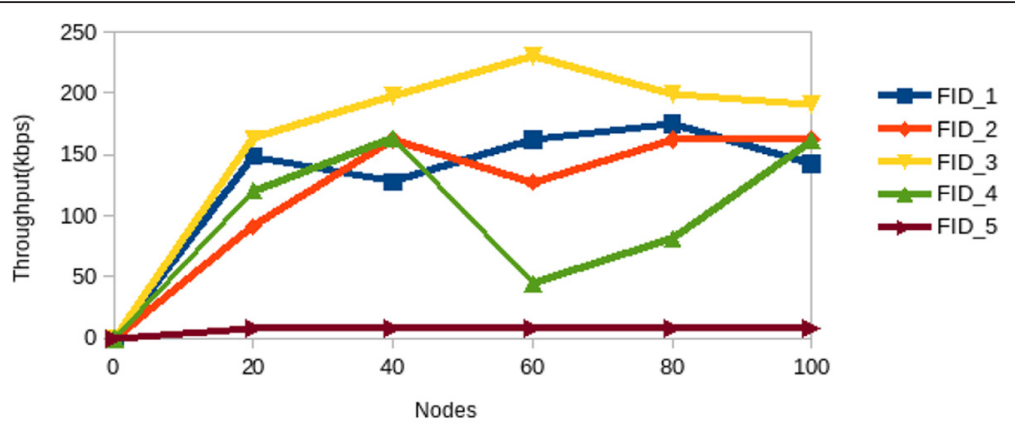

(a)

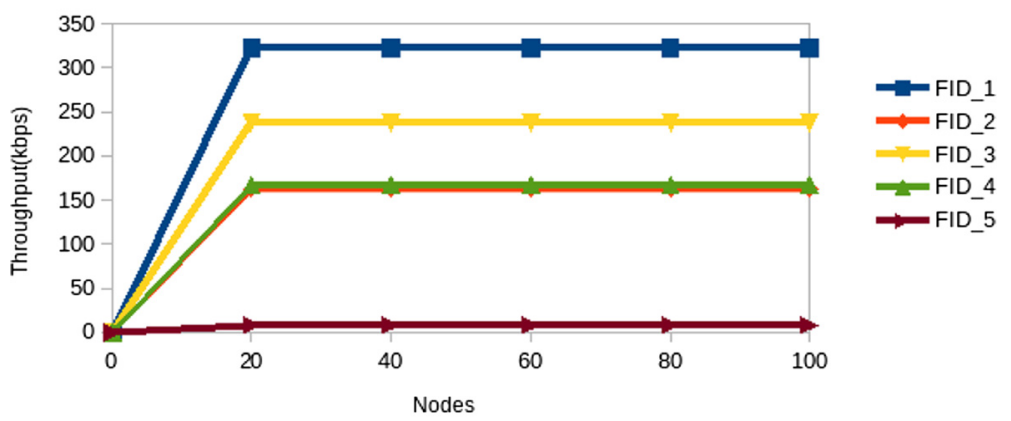

(b)

Fig. 2 Schematic representation of throughput vs number of nodes for $\mathbf{a}$ DSR and $\mathbf{b}$ PA-DSR

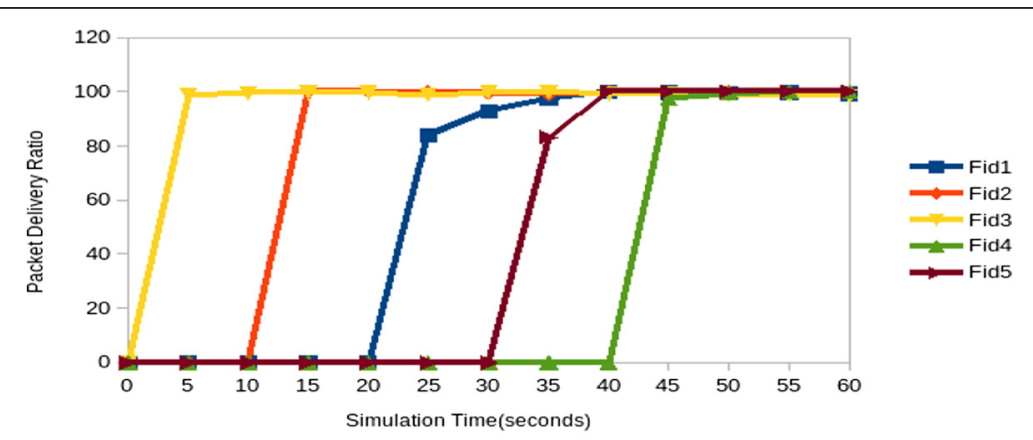

(a)



(b)

Fig. 3 Schematic representation of throughput vs number of nodes for $\mathbf{a}$ DSR and $\mathbf{b}$ PA-DSR 
Table 3 End-to-end delay for all the connections for DSR and PA-DSR protocols

\begin{tabular}{llllll}
\hline Protocol & $\begin{array}{l}\text { Flow 1 } \\
(\mathrm{ms})\end{array}$ & $\begin{array}{l}\text { Flow 2 } \\
(\mathrm{ms})\end{array}$ & $\begin{array}{l}\text { Flow 3 } \\
(\mathrm{ms})\end{array}$ & $\begin{array}{l}\text { Flow 4 } \\
(\mathrm{ms})\end{array}$ & $\begin{array}{l}\text { Flow 5 } \\
(\mathrm{ms})\end{array}$ \\
\hline DSR & 199.97 & 130.7 & 242.54 & 163.21 & 230.1 \\
PA-DSR & 15.25 & 15.13 & 10.27 & 17.22 & 21.83 \\
\hline
\end{tabular}

more accurate admission control by admitting five flows out of five. All the admitted flows meet their bandwidth requirements. The scenario indicates that the estimation that we preformed is not overestimated against the availability of bandwidth. The accuracy of the available bandwidth estimation and the admission control can be evaluated using a particular metric. This metric indicates the number of right admissions with respect to the bandwidth.

Figure 2 illustrates the variation of throughput while varying the number of nodes in the network DSR (Fig. 2a) and PA-DSR (Fig. 2b). The throughput for all the five flows are varied non-uniformly when no admission control is implemented as shown in Fig. 2a. The variation of throughput is due to underestimation of the bandwidth in the network. The throughputs of all the five flows are maintained constant while implementing the admission control. From Fig. 2b, it is investigated that the throughput is constant while increasing the number of nodes in the network.

Figure $3 \mathrm{a}, \mathrm{b}$ illustrates the effect of packet delivery ratio with respect to the simulation time for DSR and PA-DSR, respectively. The packet delivery ratio (PDR) is reached about $100 \%$, i.e., all the packets reached its destination. In Fig. 3a, the PDR of all the five connections is extended at its maximum; however, it consumes a certain amount of time to reach its maximum. It is investigated that the PDR is not reached $100 \%$ immediately once connection 3 ( $\mathrm{C} 3 \rightarrow$ 2nd second) and connection 5 (C5 $\rightarrow$ 32nd second) are initiated. The variation occurs owing to the improper bandwidth assignment. Alternatively, the PDR is attained $100 \%$ for all the connections when priority mechanism is involved as shown in Fig. 3b.

The time taken to transmit the data from source to destination of the DSR protocol is listed in Table 3. From the results, it is noticed that the average end-toend delay (EED) is about $16 \mathrm{~ms}$ for flow 1; however, for DSR, it is $253 \mathrm{~ms}$. The proposed mechanism greatly reduced the end-to-end delay than the reported one. The reduction of end-to-end delay is due to the proper assignment of bandwidth. Figure 4 shows the throughput comparison of DSR and PA-DSR of flow 3. From the simulation results, it is clearly noticed that the throughput of flow 3 is maintained constant over its simulation period for the proposed priority aware mechanism. However, for DSR, the throughput is varied abruptly.

It is hard to provide QoS guarantees when the nodes are having mobility. QoS violations can appear due to the topology changes which result either in route breakage or throughput degradation. In order to analyze the impact of network performance (flow 1 to flow 5) at mobility, the simulation is performed with 100 mobile nodes which are randomly positioned. The random way point mobility model is chosen for the motion of nodes with a speed of $20 \mathrm{~m} / \mathrm{s}$ at maximum. Five flows are considered with its designated data rates.

Figure $5 \mathrm{a}, \mathrm{b}$ depicts the effect of throughput for five different flows when DSR and PA-DSR are at the mobility speed of $20 \mathrm{~m} / \mathrm{s}$. From Fig. 5a, it is noticed that when no admission control is performed, the network becomes congested and routes are often broken involving decreased throughput flows. A certain form of admission control may therefore be necessary. From Fig. 5b, it is observed that the performance is enhanced due to the incorporation of the priority aware mechanism in DSR. The throughput of the network at mobility conditions is decreased while comparing the throughput without mobility because the mobility affects the performance of the networks. From the arrived results, it is observed that the proposed mechanism is greatly enhancing the throughput, PDR, and end-to-end delay which in turn enhances the

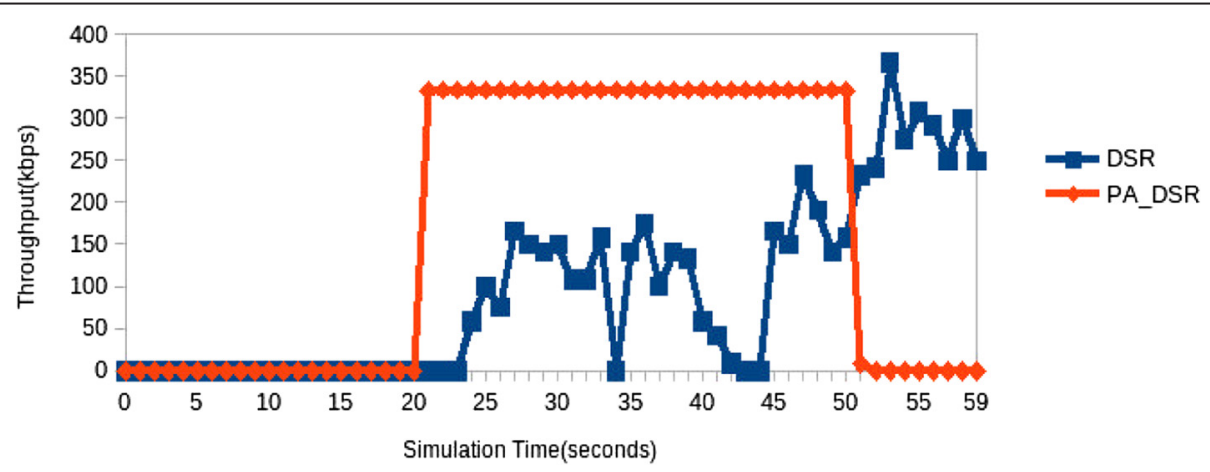

Fig. 4 Throughput comparison of DSR and PA-DSR 


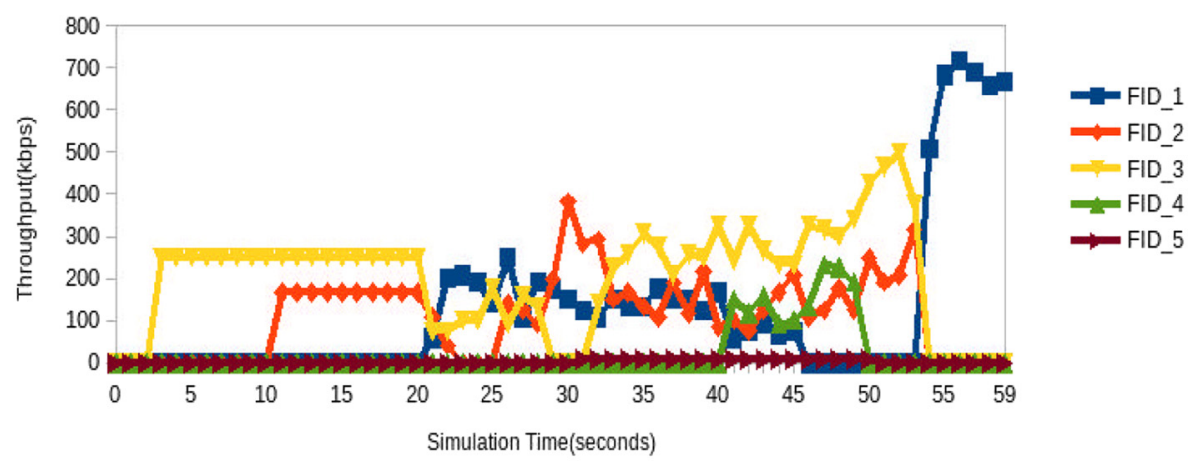

(a)

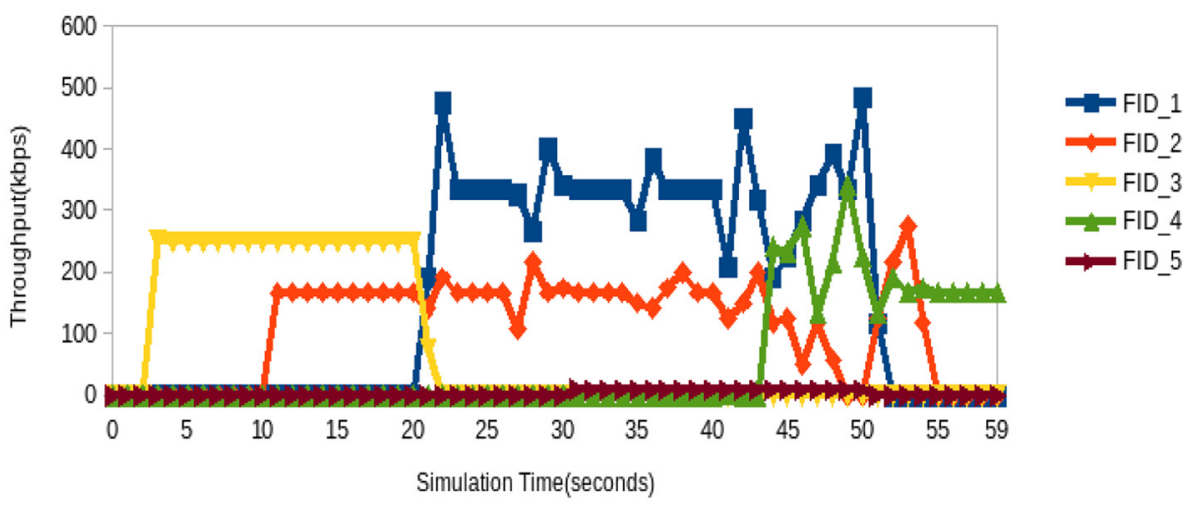

(b)

Fig. 5 Schematic representation of throughput vs number of nodes at mobility for $\mathbf{a}$ DSR and $\mathbf{b}$ PA-DSR

QoS of the network. Hence, it can be implemented for real-time applications.

\section{Conclusions}

In this paper, a priority aware dynamic source routing (PA-DSR) protocol is proposed in order to enhance the quality of service (QoS) for mobile ad hoc networks (MANETs). The priority aware mechanism is implemented through a rate cutting mechanism which is eventually executed based on their date rates. Hence, PA-DSR protocol can provide precise admission control which leads to QoS enhancement. The obtained simulation results proved significant improvement in throughput, packet delivery ratio, and end-to-end delay than existing DSR. The PA-DSR can build a QoS route from source to destination with a reserved bandwidth. It works best in small networks under low network mobility. It is hoped that in the future, ad hoc networks will emerge as an effective complement to infrastructure wired and wireless LANs and even wide area-mobile networking services.

Our future work primarily involves incorporating the proposed priority aware mechanism in other routing protocols such as AODV, AOMDV, DSDV, DYMO, and
OLSR; then evaluating its QoS parameters namely throughput, packet delivery ratio, and end-to-end delay while varying the number of nodes in the network at static and dynamic behavior; and, finally, performing comparative analysis of the simulation results obtained for conventional protocol with priority aware mechanismbased protocols, as well as implementing the priority aware mechanism in all the aforementioned protocols and comparing the QoS parameters.

\section{Competing interests}

The authors declare that they have no competing interests.

\section{Author details}

${ }^{1}$ Mount Zion College of Engineering and Technology, Pudukkottai-622507,

Tamil Nadu, India. ${ }^{2}$ Alagappa Chettiar College of Engineering and

Technology, Karaikudi-630004, Tamil Nadu, India.

Received: 11 February 2016 Accepted: 1 May 2016

Published online: 10 May 2016

References

1. S Corson, J Macker, Mobile adhoc networking (MANET): routing protocol performance issues and evaluation considerations, 1999. http://www.ietf.org/ rfc2501.txt. Accessed Jan 1999.

2. J Hoebeke, I Moerman, B Dhoedt, P Demeester, An overview of mobile ad hoc networks: application and challenges. J. Commun. Netw. 3, 60-66 (2004)

3. I Chlamtac, M Conti, JJN Lin, Mobile adhoc networking: implementation and challenges. Ad Hoc Netw. 1, 13-64 (2003) 
4. R Ananthan, J Redi, A brief overview of ad-hoc networks: challenges and directions. Communication magazine, 50th anniversary commemorative issue, 2006, pp. 20-22

5. VC Giruka, M Singhal, A self-healing on demand geographic path routing protocol for mobile ad hoc networks. Ad Hoc Netw. 2(7), 1113-1128 (2007)

6. MH Mamoun, A new proactive routing algorithm for MANETs. J. Acad. Res. 2(2), 199 (2010)

7. H Shen, L Zhao, ALERT: an anonymous location based efficient routing protocol on MANETs. IEEE Trans. Mob. Comput. 12(6), 1079-1093 (2013)

8. E Crawley, R Nair, B Rajagopalan, H Sandick, A framework for QoS based routing in the internet, August 1998, http://www.ietf.org/rfc2386.txt

9. D Wu, R Negi, Effective capacity: a wireless link mode for support of quality of service. IEEE Trans. Wirel. Commun. 2(4), 630-643 (2003)

10. S Chakrabarti, A Mishra, Quality of service challenges for wireless mobile adhoc networks. Wirel. Commun. Mob. Comput. 4(2), 129-153 (2004)

11. B Zhang, HT Mouftah, QoS routing for wireless ad-hoc networks: problems, algorithms and protocols. IEEE Commun. Mag. 43, 110-115 (2005)

12. DD Perkins, HD Hughes, A survey on quality of service for mobile ad hoc networks. Wirel. Commun. Mob. Comput. 2, 503-513 (2002)

13. SS Chaudhari, RC Biradar, Survey of bandwidth estimation techniques in communication networks. Wirel. Pers. Commun. 83(2), 1425-1476 (2015)

14. A Vasilakos, MP Saltouros, AF Atlassis, W Pedrycz, Optimizing QoS routing in hierarchical ATM networks using computational intelligence techniques. IEEE Trans. Syst. Man Cybern. Part C Appl. Rev. 33(3), 297-312 (2003)

15. S. Marwaha, D. Srinivasan, C.K. Tham, A. Vasilakos, Evolutionary fuzzy multiobjective routing for wireless mobile ad hoc networks. Evolutionary Computation, 2004. CEC2004. Congress on 2, 1964-1971, 2004

16. Y-S Yen, H-C Chao, R-S Chang, A Vasilakos, Flooding-limited and multiconstrained QOS multicast routing based on the genetic algorithm for MANETs. Math. Comput. Model. 53(11-12), 2238-2250 (2011)

17. P. Li, S. Guo, S. Yu, A. Vasilakos, CodePipe: an opportunistic feeding and routing protocol for reliable multicast with pipelined network coding. IEEE INFOCOM 2012: 100-108

18. P Li, S Guo, S Yu, A Vasilakos, Reliable multicast with pipelined network coding using opportunistic feeding and routing. IEEE Trans. Parallel Distrib. Syst. 25(12), 3264-3273 (2014)

19. C Busch, R Kannan, A Vasilakos, Approximating congestion + dilation in networks via "quality of routing" games. IEEE Trans. Comput. 61(9), 1270-1283 (2012)

20. L Liang, Y Song, H Zhang, H Ma, A Vasilakos, Physarum optimization: a biologyinspired algorithm for the Steiner tree problem in networks. IEEE Trans. Comput. 64(3), 818-832 (2015)

21. Y Song, L Liang, $\mathrm{H}$ Ma, A Vasilakos, A biology-based algorithm to minimal exposure problem of wireless sensor networks. IEEE Trans. Netw. Serv. Manag. 11(3), 417-430 (2014)

22. XM Zhang, Y Zhang, F Yan, A Vasilakos, Interference-based topology control algorithm for delay-constrained mobile ad hoc networks. IEEE Trans. Mob. Comput. 14(4), 742-754 (2015)

23. T Meng, F Wu, Y Zheng, G Chen, A Vasilakos, Spatial reusability-aware routing in multi-hop wireless networks. IEEE Trans. Comput. 65(1), 244-255 (2016)

24. C.E. Perkins, E.M. Royer, S.R. Das, Ad hoc on demand distance vector routing (DSR). Proceedings of IEEE workshop on mobile computing systems and applications 1999: 90-100

25. C Perkins, E Belding Royer, SR Das, Ad hoc on demand distance vector (DSR) routing, RFC3561, 2003. IETF MANET working group, https://www.ietf.org/rfc/ ifc4728.txt. Accessed Jul 2003.

26. R. De Renesse, M. Ghassemian, V. Friderikos, A.H. Aghvami, QoS enabled routing in mobile ad hoc networks. Proceedings of 5 th IEEE international conference on 3G mobile communication technologies 2004; 678-682.

27. $Y$ Yang, R Kravets, Contention aware admission control for ad hoc networks. IEEE Trans. Mob. Comput. 4(4), 363-377 (2005)

28. R De Renesse, M Ghassemian, V Friderikos, AH Aghvami, Adaptive admission control for ad hoc and sensor networks providing quality of service. Technical report (King College, London, 2005)

29. H Zhao, E Garcia-Palacios, J Wei, Y Xi, Accurate available bandwidth estimation in IEEE 802.11-based ad hoc networks. Comput. Commun. 32(6), 1050-1057 (2009)

30. Sabojil S. V, Akki C. B. Agent based bandwidth estimation in heterogeneous wireless networks. Proceedings of 3rd international conference on advances in recent technologies in communication and computing 2011: 256-258
31. Peng Y, Yan Z. Available bandwidth estimating method in IEEE 802.11e based mobile ad hoc network. Proceedings of 9th international conference on fuzzy systems and knowledge discovery 2012: 2138-2142

32. L Chen, WB Heinzelman, QoS-aware routing based on bandwidth estimation for mobile ad hoc networks. IEEE J. Sel. Areas Commun. 23(3), 561-572 (2005)

33. Nyambo B. M, Mugumba J, Janssens G. K. A dual bandwidth estimation method for wireless mobile ad hoc networks. AFRICON 2007; 1-6.

34. C Sarr, C Chaudet, G Chelius, IG Lassous, Bandwidth estimation for IEEE 802.11-based ad hoc networks. IEEE Trans. Mob. Comput. 7(10), $1228-1241(2008)$

\section{Submit your manuscript to a SpringerOpen ${ }^{\circ}$ journal and benefit from:}

- Convenient online submission

- Rigorous peer review

- Immediate publication on acceptance

- Open access: articles freely available online

- High visibility within the field

- Retaining the copyright to your article

Submit your next manuscript at $>$ springeropen.com 